\title{
Sustainability and innovation: a blended approach to peer mentor leader training
}

\section{Jason Hettrick}

University of Cumbria, UK

\section{Linda Moses-Allison}

University of Cumbria, UK

\begin{abstract}
Peer Assisted Study Sessions (PASS) is an established part of widening participation and retention activities at the University of Cumbria. In October 2013, the question of sustainability and growth of the scheme was addressed. The small team of PASS supervisors needed to create an effective and innovative way to meet the challenges of growing and maintaining the university's most successful peer mentoring scheme. To this end a new blended approach to PASS training was trialled in 2014. The training combined a range of face-to-face support, training and debriefs (plus online elements), via a virtual learning environment (VLE) and Facebook. Having been successful in both implementation and outcomes, this new blended approach to delivering training has now been adopted by the PASS supervisor team.
\end{abstract}

The purpose of this paper is to give an overview of the approaches and methods taken at the University of Cumbria in delivering this blended approach to PASS leader training. Initially it addresses the motivation behind the need for change, in an institutional context. It then explores some of the challenges faced during the implementation and delivery of training. Finally, it reflects on the evaluation and acknowledges future developments for leader training.

Keywords: peer mentoring; PASS; peer assisted study session; peer assisted learning; sustainability; widening participation; student support. 


\section{Introduction}

UK Higher Education (HE) institutions are seeing a growing and increasingly diverse student population as a result of the ongoing national agenda to widen participation and a greater number of non-UK students choosing to study in the UK. Over 445,000 applicants accepted a place in 2014 (UCAS, 2014). This figure included an increase in international students and those from disadvantaged and under-represented backgrounds (HEFCE, 2015; UCAS, 2014). Consequently there is a need for an inclusive, flexible, effective and sustainable method of student support that is suitable for such a diverse student body. This is directly linked to, and is reinforced in, the institution's mission statement (University of Cumbria, 2015a).

At the University of Cumbria there are a number of student support initiatives. One of these initiatives is the group peer mentoring scheme, Peer Assisted Study Sessions (PASS). The initiative was first introduced at the University of Cumbria in 2008, trialled in our Education programmes. The scheme is championed by Library and Student Services (LiSS) in collaboration with academic staff in the relevant subject disciplines. By 2013, the scheme had grown significantly; the PASS team trained 209 PASS Leaders in twelve subject disciplines, across three campuses.

\section{Background}

National Centre guidelines state that PASS leader training is traditionally delivered via two days of face-to-face delivery (University of Manchester, 2010). After reviewing the scheme, in light of expected growth and challenges for delivery, in October 2013 the PASS supervisor team discussed multiple options to ensure future sustainability. As a result it was decided to trial a combination of online and face-to-face activities. To be effective, delivery methods and approaches needed to be 'more open-ended, participative, diverse and interactive' (Sterling, 2012, p.37). It was agreed to begin trialling the blended method in early 2014. This included moving the first day of training online and the introduction of additional support via a social platform.

The decision to take the blended approach was based on a number of factors. The supervisor team was looking for a creative and innovative way in which they could deliver 
training to an increasing number of students, without jeopardising quality and experience. The scheme was continuing to grow, staff resources were stretched, and the supervisor team wanted to ensure that leaders had accessible support available. Additionally, changes would aim to address student feedback which had previously highlighted that the face-to-face training was perceived to be too long.

One of the advantages of adopting a blended approach, with the inclusion of online training, is the flexibility that it offers to students (Fleck, 2012). This is particularly important as the team wanted to put PASS Leaders in control of some of their PASS training and learning.

\section{The blended approach}

The choice of a blended approach balanced important face-to-face support, whilst allowing for the delivery of the values of PASS via an online medium. Initially, in spring 2014, prospective PASS Leaders were invited to a 60 to 90 minute workshop in their subject groups to discuss what PASS meant for them and what it would mean to be a PASS Leader. This was an opportunity to meet with their named PASS supervisor; a named contact for the subject discipline. The team introduced students to the idea that PASS should be tailored to the distinct needs of the first years within their own discipline. Tasks in this introductory training session focused on mapping the academic experience of a first year and identifying 'crunch points'. Students were also introduced to the online course and the expectations for completion.

The online element of the leader training came next. This was modelled on the, award winning, Head Start pre-entry online course offered by the university (TES, 2015; University of Cumbria, 2015b). Blackboard, the university's virtual learning environment (VLE), was deemed the natural choice for the delivery of online materials. The reason for this was twofold: students already had existing familiarity with the VLE, plus ease of tracking student progress through the course.

Articulate software was used to create the training modules. This software made it possible to combine a mix of different content types including: PASS Supervisor videos, videos of previous PASS Leaders, interactive pages and quiz elements to check 
knowledge and promote active learning. Articulate is SCORM compatible and therefore enabled tracking of student progress. A key advantage was the ability to provide instant feedback to students; an important aspect of successful online delivery (Hunt and Chalmers, 2012; Race, 2015).

The underpinning principle of the online course was to develop foundational knowledge (Amaral and Shank, 2010). The content was based on day one of training (University of Manchester, 2010). The online course introduced five main areas to the leaders: the principles of PASS; the role of the PASS leader; the importance of signposting; what happens in a PASS session; and dealing with difficult incidents. As part of the online training, Blackboard discussion boards were launched in September 2014. This aimed to provide an opportunity for students to reflect and discuss their own first year experiences. These were timed to encourage students to engage with the PASS scheme before undertaking the face-to-face training.

The next element of the training was the face to face aspect. The training day was adapted to align with the new online module. The day began with a consolidation of online training. There were then activities on Teaching vs Facilitation, Communication and Group Management, and Creating the PASS Environment - each designed to allow leaders to display what they had learnt online and put into practice some key facilitation techniques. In the latter part of the training day the focus surrounded mock sessions, enabling the leaders to prepare for their first PASS session.

There was also an optional task during the day that required leaders to engage with the new Facebook site - a social platform designed to encourage social interaction and the sharing of best practice between leaders. It also enabled the supervisor team to support new leaders with tips and session materials throughout the academic year. This approach, aligned with the use of a Blackboard module for the online training, gave the PASS Leaders the ability to re-visit information and support.

In addition to the online channels, throughout the year leaders had contact with academic coordinators and scheme supervisors during PASS debriefs and other scheduled contact. 


\section{Evaluation}

There have been varying degrees of success across the different elements of training and there are areas that will be amended and developed moving forward. After all training had taken place, leaders were asked to complete a feedback form. This allowed the team to measure how comfortable leaders felt in their role, the information that had been provided to them, and how the leaders felt about the blended approach to training. Overall, the feedback has been positive. $100 \%$ of students felt that their expectations had been met, with $86.7 \%$ stating their impression of the training was either really good or excellent. All students stated they felt they had a 'good idea' or were 'well informed' of the key areas covered in training. Additional feedback also showed that students enjoyed applying techniques through mock sessions. Surprisingly, $42 \%$ of the students still felt that the faceto-face element of the training was 'too long'.

Students have responded well to the online Blackboard module and the PASS Facebook page but, disappointingly, there was little student interaction on the discussion boards; less than $10 \%$ of the leaders engaged in this activity. PASS as a group mentoring scheme, in its very nature, is designed to be social. However, the Blackboard discussion boards were not being used in this way.

Despite the fact that the internet enables and promotes the deconstruction of social barriers, Wiley and Edwards (2002, cited in Thomas, 2010) suggest that a major challenge to online training is a tendency towards anti-social design. They argue that this is often a result of attempting to design a virtual learning environment that represents the class room. Therefore the poor engagement levels may be a result of the unfamiliarity of using Blackboard as a social platform, rather than an educational hub. For future years, the team will consider the focus of this task and repurpose the use of the discussion board.

Blackboard allowed students to revisit the underpinning theory and key principles of PASS, as and when required. However, as stated, the social interactions were limited and engagement figures were low. Higher levels of engagement on Facebook suggest that leaders have found this page useful. The number of students who engaged with Facebook was encouraging; 112 leaders initially joined the Facebook page. During the first 6-8 weeks students engaged at varying levels. Each post was 'viewed' by between $51-78 \%$ of members. Students also used the platform to discuss various subjects including useful 
techniques, questions about PASS, promotional products, signposting, and other social questions.

To look at increasing engagement for future years and to promote social interactions, a discussion activity on Facebook will be included in the online training. However, it is yet to be decided whether or not this will replace the Blackboard discussion board or be in addition to. Whilst Facebook has seen far higher levels of engagement compared to Blackboard, we cannot assume that all leaders have, or are willing to use, their personal Facebook pages for this purpose.

Some changes to the online module are being made, primarily to include more videos of experienced PASS Leaders and increase the amount of interactivity and immediate feedback given to students. Face-to-face training is also currently being updated. These changes are informed by the evaluation process that was undertaken post-training. The focus of the face-to-face training will surround the leaders' ability to practice facilitation techniques through a series of mock sessions. These activities will also be used to demonstrate and support the underpinning theory and regulations that are covered in the online training.

\section{Conclusion}

In conclusion, the blended approach should be considered a success; student feedback is positive, training is less onerous during a very busy period in the academic calendar, and students have access to an array of ongoing support. Nonetheless, ongoing, cyclical consideration and reflection are needed to ensure that this approach continues to meet the needs of all parties. Research recommends that using a variety of approaches can be useful for learners. Importantly the peer-to-peer interaction students experience through this can both improve and assist with learning during training (Howe, 2013).

After a successful trial, the blended approach will be adopted in forthcoming years. For $15 / 16$ there will be some minor adaptations. These changes will focus on integrating online and face-to-face elements. Importantly, rather than looking to measure the students' ability to recite online content, the face-to-face training will further enable students to practise and demonstrate techniques, linking the two. According to Littlejohn and Pegler (2008), rather 
than trying to seamlessly integrate online and face-to-face training, a 'wraparound' approach will help each support the other; providing that attention is spent on ensuring a coherent link between the two.

In 15/16, the PASS scheme will be delivered across 18 subject areas and five campuses. Sustaining quality whilst maintaining growth is still a priority for the supervisor team and placing part of the training online will enable the team to dedicate more time to planning and supporting students from a wider range of programmes. Ultimately, it is hoped that the number of leaders trained and the number of students that are likely to participate in the PASS scheme will increase.

This case study demonstrates that adopting a blended approach to PASS leader training and development can be successful. This method can provide an effective way of meeting the requirements of all stakeholders and assist in ensuring a continued and sustainable student support scheme.

\section{References}

Amaral, K.E. and Shank, J.D. (2010) 'Enhancing student learning and retention with blended learning class guides', Educause Quarterly, 33(4), p. 16.

Fleck, J. (2012) 'Blended learning and learning communities: opportunities and challenges', The Journal of Management Development, 31(4), pp. 398-411.

HEFCE (2015) Higher education in England 2015. Key facts. Available at: http://www.hefce.ac.uk/analysis/HEENG/ (Accessed: 5 July 2015).

Howe, C. (2013) 'Scaffolding in context: peer interaction and abstract learning', Journal of Learning, Culture and Social Interaction, 2(1), pp. 3-10.

Hunt, L. and Chalmers, D. (eds.) (2012) University teaching in focus. A learning-centred approach. London: Routledge.

Littlejohn, A. and Pegler, C. (2008) Preparing for blended E-learning. London: Routledge. 
Race, P. (2015) The lecturer's toolkit. $4^{\text {th }}$ edn. London: Routledge.

Sterling, S. (2012) The future fit framework: an introductory guide to teaching and learning for sustainability in H.E. York: Higher Education Academy. Available at: https://www.heacademy.ac.uk/sites/default/files/future fit 270412 1435.pdf (Accessed: 9 August 2015).

TES (2015) Previous winners. Available at:

http://www.thelmawards.co.uk/thelma2015/previous-winners (Accessed: 11 August 2015).

Thomas, H. (2010) 'Learning spaces, learning environments and the dis'placement' of learning', British Journal of Educational Technology, 41(3) pp. 502-511.

UCAS (2014) Undergraduate 2014 end of cycle report. Available at: https://www.ucas.com/corporate/data-and-analysis/analysis-reports (Accessed: 5 July 2015).

University of Cumbria (2015a) Corporate strategy 2012-2017. Available at: http://www.cumbria.ac.uk/Public/VCO/Documents/Communications/Publications/Co rporate-Strategy-2012-17.pdf (Accessed: 11 August 2015)

University of Cumbria (2015b) Head start. Available at:

http://www.cumbria.ac.uk/Courses/Subjects/BusinessComputing/CPD/HeadStart.as px (Accessed: 30 June 2015).

University of Manchester (2010) ‘PASS folder@ PASS/ SI Supervisor Training May 2012. Peer Assisted Study Sessions. The University of Manchester. Unpublished. 


\section{Author details}

Jason Hettrick is a WP Learning Enhancement and Retention Adviser at the University of Cumbria. In the early stages of his career in academia, his current focus surrounds academic skills development, student support and peer mentoring. Research interests include peer support, pedagogical practices and retention initiatives.

Linda Moses-Allison is a Library and Academic Adviser at the University of Cumbria. She has worked in various roles within library settings, all with a student support focus. Linda led the project to develop PASS Leader training online at the University of Cumbria. Her research interests include online learning within student services and Peer mentoring. 